\title{
Face recognition based on Radon transform
}

\author{
Meixia Yang ${ }^{1, a}$, Zhuming $\mathrm{CaO}^{2, \mathrm{~b}}$ \\ ${ }^{1}$ The department of information engineering Tianjin Modern Vocational Technology CollegeTianjin, \\ Chain \\ ${ }^{2}$ Mechanical Engineering college Beijing Electronic Science and technology Vocational Technology \\ CollegeTianjin, Chain

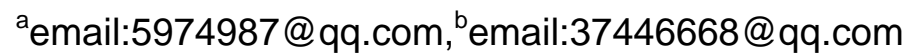

Keywords: face recognition; complex conditions; recognition algorithm; Radon transform; wavelet transform .

\begin{abstract}
Aiming at the problem that most of the face recognition algorithms can not overcome the complex conditions such as illumination, expression and occlusion, a face recognition algorithm based on Radon transform is proposed. In this algorithm, the face image is pre processed by using the normalized algorithm and wavelet transform. Then the Radon transform is used to extract the invariant features.
\end{abstract}

\section{INTRODUCTION}

Face recognition algorithm in recent years has been rapid development, and some face the algorithm can overcome the interference of some external conditions to a certain extent, and achieved a higher recognition rate, but these algorithms substantially only for lighting, posture, facial expressions, research occlusion interference conditions in a way, more complex conditions under interference low recognition rate. For more complex conditions present a certain universal algorithm is relatively small, mainly the use of three-dimensional face modeling method, but this method has a major problem is the excessive computing. Based on the above considerations, this paper attempts to use conversion as face recognition feature extraction algorithm, transformation [12] compared with SVD, PCA, LDA, ICA and other feature extraction algorithm has better lighting insensitivity, translation of, scalability, and noise immunity. Currently, the conversion is effectively applied to the target linear object detection [3], the medical image enhancement [4], image retrieval [5], handwritten signature recognition [6] and so on. In recent years, transform in face recognition applications have published literature [7-8], but is still in its infancy. So hope to use text features to transform and overcome illumination and expression, blocking effect on face recognition, face recognition algorithm so that a certain universality in the many complex environmental conditions.

\section{RADON TRANSFORM}

Radon transform is a German mathematician J.Radon proposed to determine a mathematical function of the method by integrating all functions in the plane of the line. In 1966, Luwing proposed Radon in n dimensional Euclidean geometric space transform so that the Radon transform has been widely used in pattern recognition and image processing field.

Transformation is the essence of the line and the point of the dual transformation problem, put an image as a function, the function converts a straight line along a plane containing a family of functions on the line integrals. Simply put, it is the X,Y coordinate plane two-dimensional image in a line mapped to a point in space. Mathematical transformation is described as:

$\mathrm{P}(\mathrm{r}, \theta)=\mathrm{R}(\mathrm{r}, \theta)\{f(x, y)\}=\iint f(x, y) \delta(\mathrm{r}-x \cos \theta-y \sin \theta) d x d y(1)$

Among them, $\delta$ on behalf of a unit pulse function, expressed in the form of:

$\delta(\mathrm{K})= \begin{cases}1 & \mathrm{~K}=0 \\ 0 & \mathrm{~K} \neq 0\end{cases}$ 
$f(x, y)$ It represents the gray values in the image $(x, y)$ at the pixel, $r$ represents the origin of coordinates to the vertical distance from the line to be detected, $\theta$ is the angle between the coordinate axis $\mathrm{x} \mathrm{r}$, the function $\delta$ is a straight line $\mathrm{r}-x \cos \theta-y \sin \theta$ integrated, in order to obtain at any point $(r, \theta), f(x, y)$ function along the straight line projection value $P(r, \theta)$.

Figure 1 is an AR face database face samples in different states(Normal state, the expression changes, illumination changes, occlusion change) Characteristics of Radon, Radon transform angle, respectively as 18 degrees, 36 degrees, 90 degrees. You can see in the figure, Face the same angle under different conditions of interference, Radon Basic characteristics do not change, indicating that the feature space for face images of different interference has certain invariance.
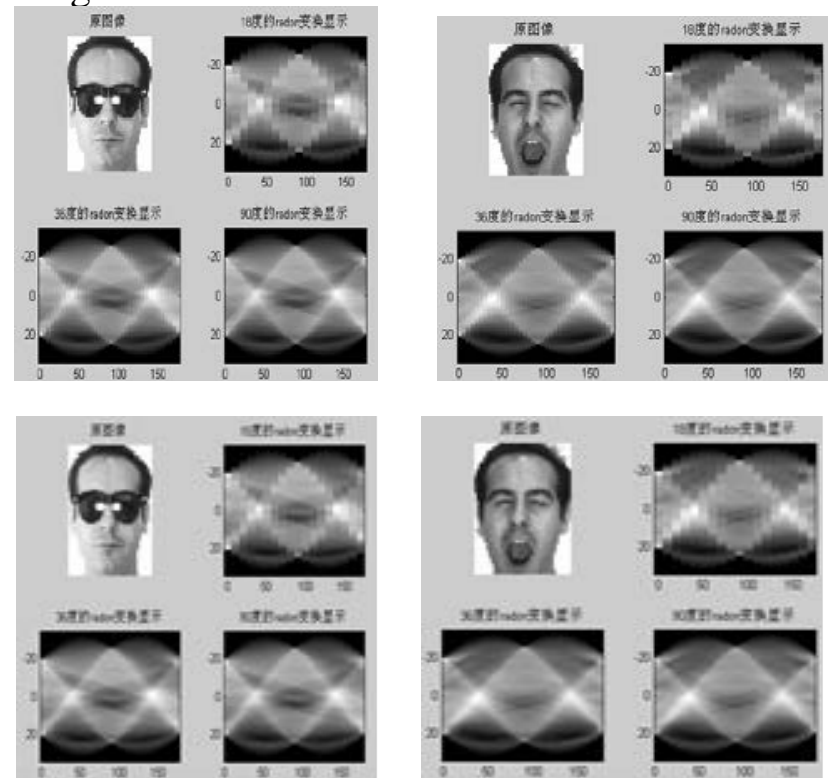

Figure 1 Radon characteristics of face image with different views under different conditions

\section{DIMENSIONAL DISCRETE WAVELET TRANSFORM (2D-DCT)}

In order to light one of the normalized image after further compression, reducing the subsequent facial feature extraction computation, two-dimensional discrete wavelet transform (2D-DWT) face image after illumination normalization transform.One-dimensional mathematical description of continuous wavelet transform is:

$$
W(a, b)=\int_{-\infty}^{\infty} f(t) \frac{1}{\sqrt{a}} \mu\left(\frac{t-b}{a}\right) d t
$$

Wherein, a representative of the wavelet transform scale factor, b representative of a shift factor. The continuous one-dimensional continuous wavelet scale factor and translation factor according to powers of two discrete process can get a one-dimensional discrete wavelet. The definition of a onedimensional discrete wavelet is:

$$
\left\{f, \quad \psi_{\mathrm{m}, \mathrm{n}}\right\}=a^{-\frac{\mathrm{m}}{2}} \int_{-\infty}^{\infty} f(t) \psi\left(a^{-m} t-n b\right) d t
$$

Dimensional discrete wavelet transform (2D-DWT) is an extended form the basis of the onedimensional discrete wavelet transform on, its essence is a two-dimensional image signal on different scales finer decomposition. An image after the image of two-dimensional discrete wavelet transform layer is divided into four sub-bands: LL, LH, HL, HH. LL layer which reflects the approximate characteristics of the image, while the other three sub-band component band reflects the image details. The following figure shows AR face database through a face image renderings dimensional discrete wavelet transform and wavelet Process diagram. 


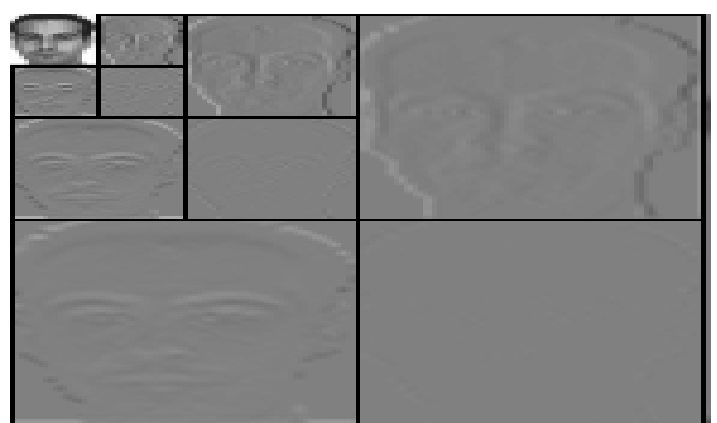

Figure 2 2D-DWT decomposition effect

\section{FACE IMAGE ILLUMINATION NORMALIZATION PREPROCESSING}

Because with a human face images in different lighting conditions using the same kinds of feature extraction algorithm acquired feature will appear completely different situation, In order to make an image of the face lighting effects to reach consensus, we use a normalized illumination method light pretreatment. Specific implementation process is as follows:

a) illumination normalization algorithm The first step is to use the literature [9-10] Retinex algorithm described in the multiscale Retinex algorithm, the main purpose is to remove part of the shadow phenomenon appearing in the face image, a face image control overall brightness. The mathematical description is:

$$
\left\{\begin{array}{l}
R_{M S R_{i}}=\sum_{n=1}^{N} \omega_{n} R_{n_{i}} \\
R_{n_{i}}=\log I_{i}(x, y)-\log \left[F_{n}(x, y) * I_{n}(x, y)\right] \\
F_{n}(x, y)=K_{n} \cdot e^{-\left(x^{2}+y^{2} / c_{n}^{2}\right)} \\
\iint F_{n}(x, y) d x d y=1
\end{array}\right.
$$

b) illumination normalization algorithm of the second step Gaussian differential filter,, Its purpose is to remove the edge after illumination face image becomes smooth, to remove noise effects. The mathematical expression is:

$\mathrm{G}(\mathrm{s})=\mathbf{A}_{1} \mathrm{e}^{-\mathrm{s} / 2 \delta_{1}^{2}}-\mathbf{A}_{2} \mathbf{e}^{-\mathrm{s} / 2 \delta_{2}^{2}}$

c) The final step illumination normalization algorithm is the use of contrast equalization algorithm, which aims to face images of gray-level readjusted to achieve the facialimage contrast and brightness to make a standardized process. The algorithm is as follows:

$$
\frac{I(x, y)}{\left(\operatorname{mean}\left(\min \left(\tau,\left|I\left(x^{\prime}, y^{\prime}\right)\right|\right)^{a}\right)\right)^{1 / a}} \longrightarrow I(x, y)
$$

Rendering preprocessed shown in Figure 3

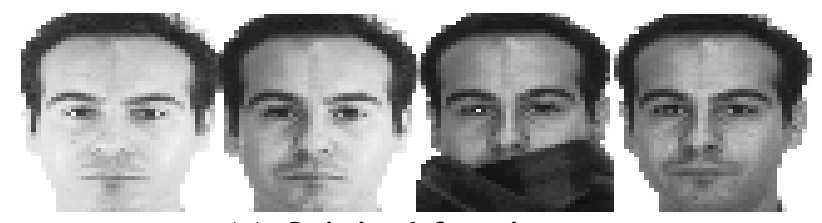

(a) Original face image

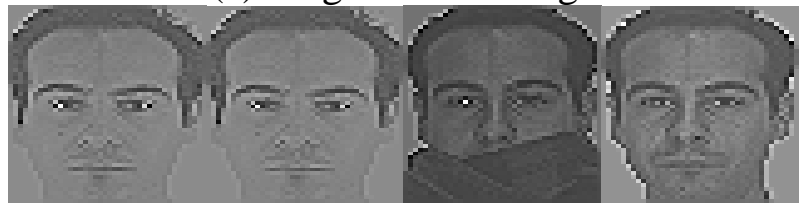

(b) Effect after light normalization

Figure 3 Effect after light normalization of face image 


\section{FACE RECognition BASEd on RAdon Transform}

Application of Radon transform of specific recognition algorithm procedure is as follows:

Step 1: the use of Section 3 of the illumination normalization algorithm, the different light levels normalized face image processing, People face image of gray scale to reach a uniform standard.

Step 2: After using the Radon transform processing Step 1 person Face image feature extraction. This feature has a rotational invariance, invariance and a certain proportion of noise immunity.

Step 3: using principal component analysis (PCA) of the Radon transform of characteristic dimension reduction process to reduce the subsequent recognition of computation.

Step 4:Euclidean distance calculation to be recognized face image feature vector feature vector library and training between the human face image.

\section{RESULTS OF EXPERIMENTS AND FACE RECOGNITION.}

\section{A. Select the face database}

Select one of the AR face database subset gray section(AR_ Gra y), the library contains 120 individual face of 3120 face images, in which each person 26 images. This image contains 26 changes in the expression of the human face, the light changes, and occlusion of interference, people involved face image change is very comprehensive, very suitable for testing a variety of face recognition algorithms under complicated conditions change face recognition performance.Some people face database sample shown in Figure 4.

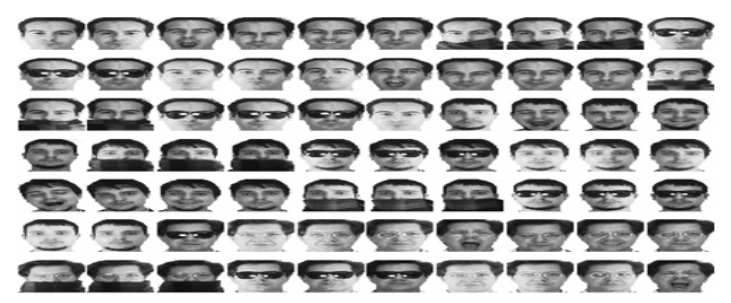

Figure 4 Samples of AR face library in experimnet

B. Experimental results and analysis

In the experiment, randomly selected 13 images each person as training set, in addition to 13 images as a test set. In order to facilitate the subsequent recognition will cut all images to $40 \times 50$ size. In comparison algorithm selection using the PCA, KPCA, LDA, GFC, LPP five classic algorithm and the proposed algorithm is compared, in order to achieve the principle of fairness, Five kinds of classic face algorithms are used in the pretreatment phase histogram equalization algorithm for face image preprocessing.

Recognition rate algorithms are shown in Table 1.

Table 1.Recognition rate of face recognition algorithm in AR library

\begin{tabular}{|r|l|l|l|l|l|l|}
\hline ALGORITHM & PCA & KPCA & LDA & CFG & LPP & This paper \\
\hline $\begin{array}{c}\text { RECOGNITIO } \\
\text { N RATE (\%) }\end{array}$ & 54.13 & 58.29 & 76.38 & 86.52 & 71.32 & 95.28 \\
\hline
\end{tabular}

Can be seen from Table 1, the proposed algorithm has a certain advantage in the recognition rate compared with the five classical algorithm, can achieve $95.28 \%$ higher recognition rate. This is mainly because the algorithm used in the pretreatment stage illumination normalization algorithm can be better. Removing the effect of light on the human face of the image, so that people affected by different light face image to achieve an identical grayscale range, feature extraction stage in the use of Radon transform to extract invariant features of face images. The illumination invariant features of the human face, facial expression, occlusion change has better robustness. Therefore, the proposed face recognition algorithm recognition algorithm than the traditional recognition disturbance in a variety of complex conditions more advantages. 


\section{RESULTS OF EXPERIMENTS AND FACE RECOGNITION.}

In this paper, based on facial Radon transform algorithm, the algorithm first face image illumination normalization pretreatment, and then using the Radon transform to extract face feature space, and the use of wavelet transform and PCA feature space for further dimensionality reduction processing, and finally two-dimensional nearest neighbor classifier complete recognition.We verify the algorithm illumination, expression and occlusion of recognition under the condition of interference with a certain robustness and universality in experiments in AR face database.

\section{REFERENCES}

[1] Zhang Junhua, Yang Cun, Xu Hui, et al. High Reso-lution Parabolic Radon Transform by Frquency DomainMethod for Eliminating Multiple [C] // Proc of Cogresson Image and Signal Processing. Sanya, China, 2008:233-237.

[2]Trad D O, Ulry ch T J, Sacchi M D.Accurate Interpo-lation with High-Resolution TimeVariant RadonTransform[J]. Geophysics,2002,67(2):644-656.

[3]Terrades O R,Valveny E.Radon Transform for LinealSymbol [C] // Proc of the 7 th International Conference onDocument Analysis and Recogni-tion. Edinburgh,Scotland,2003,I:195-199.

[4]TIAN Yun, XU Liang, ZHANG Qi, et al . Based on Radon transform medical chart as enhancement method[j]. Journal of Microelectronics and Computers, 2003,23(5):12-14.

[5] Gui Peiling, Li Junhong, Pan Quan, et al. Rotation and Scaling Invariant Texture Classification Based on Radon Transformand Multi-scale Analysis [j] . Pattern Recognition Letters,2006,27(5):408-413.

[6]Maiorana E, Campisi P, Neri A. Biometric SignatureAuthentication Using Radon Transform Based water-marking Techniques[C] // Proc of the Biometrics Sym-posium.Baltinore,USA,2007:16.

[7]Dattatray V. Jadhav, Raghunath S.Holambe. Radon and discrete cosine transforms based feature extreaction and dimensioality reduction approach for face recogni-tion[J]. Signal Processing,2008,88(10):2604-2609.

[8]GAN Junying, HE Sibin. Nonlinear Radon transform And its application in face recognition[J]. Journal of ar- tificial intelligence and pattern recognition, 2011,24(3):406-410.

[9]Tan X, Triggs B. Enhancced local texture feature sets for face recongnition under difficult lighting conditions[J]. IEEE Trans on Image Processing, 2010, 19(6) :1635-1650.

[10]ZHANG Yi, XIONG Fei, ZHANG Guilin. A Prepro-cessing Algorithm for Illimination Invariant Face Rec-ognition[J]. Journal of Image and Graphics, 2008, 13(9):1707-1712. 